EXTENDED REPORT

\title{
Femorotibial and patellar cartilage loss in patients prior to total knee arthroplasty, heterogeneity, and correlation with alignment of the knee
}

\author{
R von Eisenhart-Rothe, H Graichen, M Hudelmaier, T Vogl, L Sharma, F Eckstein
}

Ann Rheum Dis 2006;65:69-73. doi: 10.1136/ard.2005.038869

See end of article for authors' affiliations

Correspondence to:

Prof Dr med Felix Eckstein Institute for Anatomy and Musculoskeletal Research, Paracelsus Private Medical University, Strubergasse 21, A 5020 Salzburg, Austria; felix.eckstein@ pmu.ac.at

Accepted 14 June 2005 Published Online First 23 June 2005

\begin{abstract}
Objective: To analyse tibial, femoral, and patellar cartilage loss in patients prior to total knee arthroplasty (TKA), and its correlation with alignment of the knee.

Methods: 26 patients (aged 58 to 86 years) with a clinical indication for TKA were investigated. Quantitative end points of cartilage morphology (T scores for cartilage volume normalised to total subchondral bone area) were determined from coronal and axial magnetic resonance image data, using proprietary software. The static alignment of the knee was determined from standing full limb radiographs. Results: The magnitude of cartilage loss (T score of normalised cartilage volume) was highly variable within the knee, correlation coefficients ranging from $r=0.17$ to 0.51 between cartilage plates. The correlation of cartilage loss with static alignment of the knee (as a continuous variable) was $r=-0.52$ $(p<0.05)$ for the medial tibia, -0.38 (not significant) for the medial femur, $+0.76(p<0.001)$ for the lateral tibia, +0.31 (not significant) for the lateral femur, and -0.09 for the patella. When analysing alignment independent of direction (valgus or varus), the correlation for the patella increased to $r=0.30$, but remained non-significant.

Conclusions: Cartilage loss was highly variable among patients and among cartilage plates before knee arthroplasty. Its correlation with alignment was stronger for the tibia than for the femur. There was some evidence for an association of alignment and patellar cartilage loss. These findings stimulate further research on the mechanism and cause-effect relation of alignment and knee osteoarthritis using quantitative magnetic resonance imaging technology.
\end{abstract}

O steoarthritis represents an outstanding burden on the quality of life of elderly people and on the economics of today's health care systems. ${ }^{12}$ The disease is characterised by a loss of articular cartilage and changes in non-cartilage tissues, such as bone, ligaments, menisci and synovium. As cartilage tissue could not be quantified by noninvasive means until recently, information on cartilage loss in osteoarthritis has been sparse and has been based primarily on indirect evidence from joint space width measurements in radiographs. Several studies have now established that quantitative magnetic resonance (MR) imaging allows measurement of the morphology of articular cartilage with high accuracy and precision ${ }^{3-6}$ if appropriate imaging protocols and image analysis tools are used. Adequate accuracy and precision has also recently been confirmed for patients before knee arthroplasty. ${ }^{4}$

In cross sectional studies, cartilage volume has been shown to be a relatively insensitive outcome measure of osteoarthritis, owing to confounding by bone size. ${ }^{7}$ Considerable improvements in the discrimination between healthy subjects and patients with osteoarthritis (T scores) have, however, been achieved by normalising cartilage volume to the total subchondral bone area of cartilage plates, including cartilaginous and denuded surface areas. ${ }^{7}$

Epidemiological research has identified numerous risk factors associated with osteoarthritis in various joints of the body. ${ }^{8-10}$ In the knee, malalignment (valgus/varus) appears to be associated with a higher prevalence and progression of osteoarthritic changes in the relevant compartment, ${ }^{11}{ }^{12}$ in particular in association with obesity. ${ }^{13}$ This probably reflects the alteration in load distribution in the knee, with higher loads being transmitted across the medial femorotibial compartment in varus malalignment, and higher loads across the lateral femorotibial compartment in valgus malalignment. ${ }^{13}$ On the basis of measurements of joint space width in radiographs, patients with varus osteoarthritis appear to show a fourfold greater rate of progression of cartilage loss in the medial femorotibial compartment, and patients with valgus osteoarthritis a fivefold greater progression rate in the lateral femorotibial compartment. ${ }^{11}$ A recent MR imaging study has found greater cartilage volume loss in the medial femorotibial compartments of patients with moderate symptomatic osteoarthritis and varus malalignment, and a greater loss in the lateral compartment in patients with valgus malalignment. ${ }^{12}$ There has, however, been no information on the extent to which patellar cartilage loss is associated with alignment of the knee. Whereas varus malalignment may increase the loading on the medial patellar facet, valgus malalignment may increase the mechanical stress at the lateral facet.

The objective of this cross sectional study was to use quantitative MR imaging for analysing cartilage loss (T scores) of tibial, femoral, and patellar cartilage in patients with advanced osteoarthritis, before knee arthroplasty, and to relate cartilage loss in each plate to static alignment of the knee. We specifically addressed the following questions. First, what is the magnitude of patellar, tibial, and femoral cartilage loss with and without normalisation to total bone

Abbreviations: $\mathrm{BMI}$, body mass index; $\mathrm{CLF}$, lateral aspect of the femur; $C M F$, medial aspect of the femur; $L T$, lateral tibia; $M T$, medial tibia; $t A B$, total subchondral bone area of the cartilage plates; ThCCAB.max, maximum thickness of the cartilage; ThCCAB.me, mean thickness of the cartilage, as an average of the cartilage covered area of bone; TKA, total knee arthroplasty; $V C$, cartilage volume; $V C t A B$, cartilage volume normalised to body height, body weight, and $t A B$ 
area, body weight or body height in patients before TKA? Second, how strongly are tibial, femoral, and patellar cartilage loss correlated among each other in advanced osteoarthritis? And third, to what extent is this cartilage loss associated with valgus/varus malalignment of the knee, with static alignment being expressed as a continuous variable?

\section{METHODS}

Informed written consent was obtained from the patients, and the study protocol was ratified by the local ethic committee (University of Frankurt, Germany)

The study involved 26 patients (mean (SD) age 70.4 (7.6) years, range 58 to 86 ; six men, 20 women) with a clinical indication for TKA. Mean height was 164 (6) $\mathrm{cm}$ and 175 (14) $\mathrm{cm}$ in women and men respectively, body weight was 82.3 (12.9) kg and $97.2(22.6) \mathrm{kg}$, and body mass index was $30.6(4.6) \mathrm{kg} / \mathrm{cm}^{2}$ and $31.5(3.2) \mathrm{kg} / \mathrm{cm}^{2}$. MR imaging was undertaken in vivo before TKA, as described previously. ${ }^{6}$ In brief, coronal datasets for the femorotibial compartments and axial scans of the patellar cartilage were acquired for quantitative indices of cartilage morphology, as these have been shown to yield high accuracy, ${ }^{4}{ }^{6}$ test-retest precision, ${ }^{4}{ }^{14}$ and sensitivity to change in longitudinal studies ${ }^{15}{ }^{16}$. Analysis of the patella (P) and the medial and lateral tibia (MT and LT) involved the entire cartilage plates; analysis in the femur involved the central region of the medial and lateral aspect of the femur (CMF and CLF, respectively) - that is, the anterior aspect of the femoral condyles, ${ }^{14-16}$ a region that represents its weight bearing portion in the extended and slightly flexed position of the knee.

The following quantitative end points of cartilage morphology were determined using proprietary software ${ }^{17-19}$ : total volume of the cartilage (VC); mean thickness of the cartilage, as an average of the cartilage covered area of bone and not accounting for denuded areas yielding $0 \mathrm{~mm}$ cartilage thickness (ThCcAB.me); the maximum thickness of the cartilage (ThCcAB.max); and the total subchondral bone area of the cartilage plates, including cartilaginous and denuded areas, but not peripheral osteophytes ${ }^{6}$ (tAB). Because a previous study has indicated that cartilage volume provides insufficient discrimination between healthy subjects and osteoarthritic patients owing to confounding of bone size, we normalised cartilage volume to body height, body weight, and $\mathrm{tAB}(\mathrm{VC} / \mathrm{tAB}) .^{7}$ Values were compared with a reference database that involved 50 young healthy subjects aged 19 to 35 years (23 women aged 25.7 (3.6) years; 27 men aged 26.0 (4.0) years) without symptoms or signs of osteoarthritis, history of knee pain, trauma, surgery, ligament and meniscal injury, or other diseases of the musculoskeletal system.

T scores for cartilage morphology (difference between patient value and mean value in young healthy subjects of the same sex, divided by the standard deviation in healthy subjects of the same sex) were computed for all cartilage
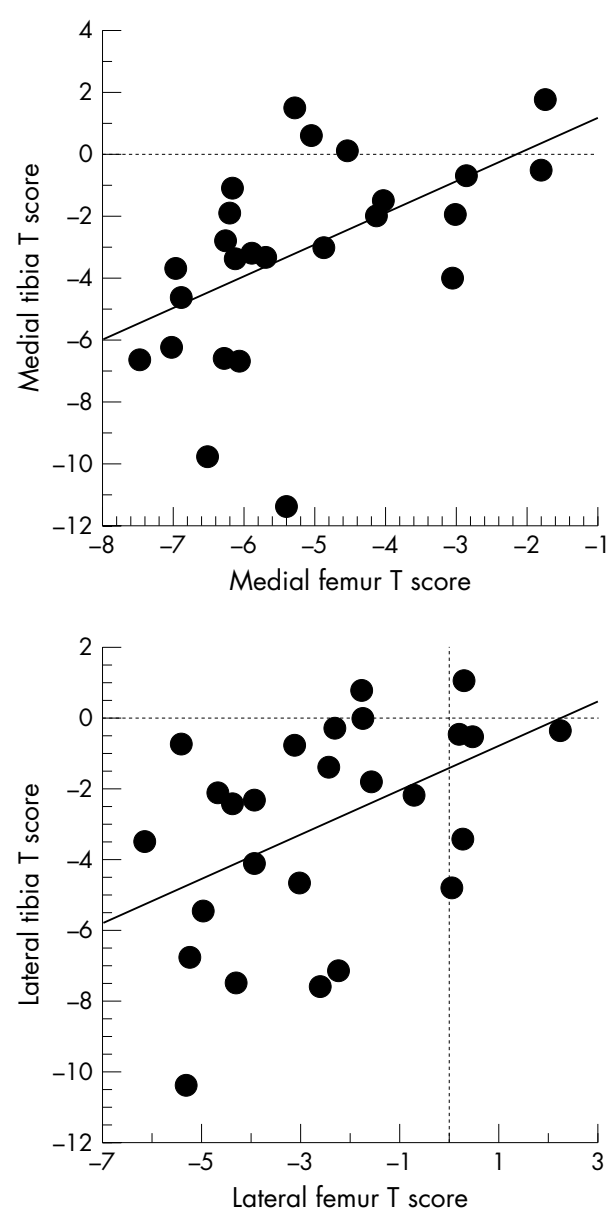

Figure 1 Bivariate scattergram showing the correlation between T scores for cartilage volume normalised to total subchondral bone area in the medial tibia and medial femur, and in the lateral tibia and lateral femur, respectively.

plates, as described previously. ${ }^{7}$ The association between patellar, tibial, and femoral $\mathrm{T}$ scores, and that between $\mathrm{T}$ scores and the static alignment of the knee, was determined by linear regression analysis. An average medial femorotibial $\mathrm{T}$ score was computed by averaging the $\mathrm{T}$ scores in the medial tibia and medial femoral condyle, and the same was done laterally.

The static alignment of the knee was determined from standing full limb radiographs in 22 of the 26 patients. To this end, we determined the medial angle between an axis drawn through the middle of the femoral head and intercondylar notch of the distal femur, and an axis through the middle of the intercondylar area of the proximal tibia and

Table 1 T scores for quantitative cartilage parameters in advanced knee osteoarthritis

\begin{tabular}{llllll}
\hline & MT & cMF & LT & cLF & P \\
\hline VC & -2.2 & -2.4 & -2.0 & -0.5 & -2.6 \\
ThCcAB.me & -1.4 & -4.0 & -2.3 & -1.6 & -3.1 \\
ThCcAB.max & -0.6 & -2.8 & -2.3 & -0.8 & -2.4 \\
VC/weight & -2.9 & -2.8 & -3.1 & -1.2 & -2.4 \\
VC/height & -2.1 & -2.3 & -2.0 & -0.4 & -2.4 \\
VC/ $+A B$ & -3.2 & -5.2 & -3.0 & -2.6 & -3.2 \\
\hline
\end{tabular}

$\mathrm{CLF}$, central lateral femur; $\mathrm{CMF}$, central medial femur = anterior part of the medial femoral condyle; LT, lateral tibia; MT, medial tibia; $P$, patella; $t A B$, total area of subchondral bone, including cartilaginous and denuded areas but excluding peripheral osteophytes; ThCcAB.max, maximum cartilage thickness; ThCcAB.me, mean thickness of the cartilage in cartilage covered areas, but not accounting for denuded areas as being $0 \mathrm{~mm}$ cartilage thickness; VC, total volume of the cartilage. 
Table 2 Correlation of T scores (VC/ $\mathrm{tAB}$ ) in knee cartilage plates in advanced osteoarthritis

\begin{tabular}{lccll}
\hline & cMF & LT & cLF & P \\
\hline MT & $+0.51^{* *}$ & -0.30 & +0.23 & +0.17 \\
CMF & - & -0.17 & +0.24 & +0.26 \\
LT & - & - & $+0.46^{*}$ & +0.29 \\
cLF & - & - & - & +0.28 \\
\hline
\end{tabular}

${ }^{*} \mathrm{p}<0.05 ;{ }^{* *} \mathrm{p}<0.01$.

$\mathrm{cLF}$, central lateral femur; $\mathrm{CMF}$, central medial femur = anterior part of the medial femoral condyle; LT, lateral tibia; MT, medial tibia; P, patella.

the middle of the talocrural joint. Fourteen patients showed varus malalignment (range $+4^{\circ}$ to $+14^{\circ}$ ), six had valgus malalignment (range $-3^{\circ}$ to $-19^{\circ}$ ), and two had neutral alignment $\left(0^{\circ}\right)$.

\section{RESULTS}

The $\mathrm{T}$ scores of all cartilage plates were higher for cartilage volume (VC) normalised to total area of subchondral bone (tAB) than for VC alone or VC normalised to body weight and height (table 1). These findings show that normalisation to $\mathrm{tAB}$ is the most effective means of evaluating cartilage volume loss in cross sectional studies. Among the knee cartilage plates patellar T scores were highest before normalisation to $\mathrm{tAB}$, but the scores were highest in cMF after normalisation. T scores were higher for CMF than for MT and as high for CLF as for LT, suggesting that the femoral region of interest (the anterior condyle) is a region sensitive to change in osteoarthritis (table 1). The combined medial $\mathrm{T}$ score $(\mathrm{VC} / \mathrm{tAB})$ in the medial femorotibial compartment was $-4.2(2.1)$, the combined lateral femorotibial $\mathrm{T}$ score $-2.8(2.2)$, and the patellar $\mathrm{T}$ score -3.2 (1.4) in the TKA patients (mean (SD)).

The correlation of cartilage loss ( $\mathrm{T}$ scores for $\mathrm{VC} / \mathrm{tAB}$ ) among the cartilage plates of the knee was weak (table 2). Only the correlation coefficients between the medial tibia and medial femur, and between the lateral tibia and lateral femur, reached statistical significance (fig 1). Even the

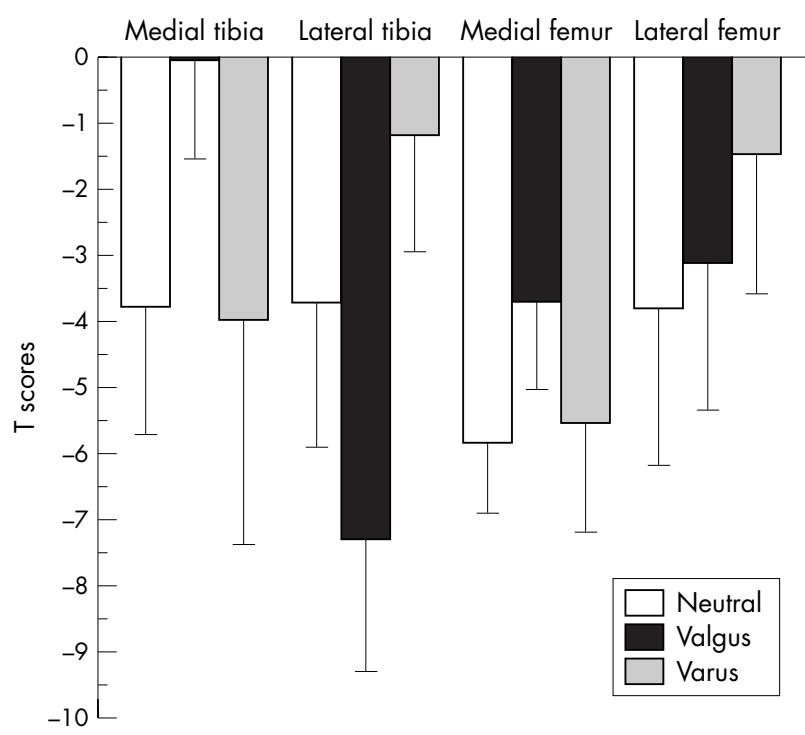

Figure 2 Bar graph showing T scores for cartilage volume normalised to total subchondral bone area in the medial tibia, medial femur, lateral tibia, and lateral femur for patients with varus osteoarthritis $(n=14)$, those with neutral alignment of the knee $(n=2)$, and those with valgus osteoarthritis $(n=6)$. Error bars show the onefold standard deviation. correlation between femoral and tibial cartilage loss of the same compartment was only moderate $(r=0.51$ and 0.46 , respectively), suggesting that the proportion of femoral and tibial cartilage loss is highly variable in advanced femorotibial osteoarthritis.

Figure 2 summarises $\mathrm{T}$ scores for patients with valgus malalignment $(n=14)$, varus malalignment $(n=6)$, and those with a neutral knee axis $(n=2)$. When taking the knee axis as a continuous variable (negative values = valgus, positive values $=$ varus) we found a moderate association of the (average) medial T scores $(r=-0.54 ; \mathrm{p}<0.01)$ and lateral T scores $(r=+0.66 ; \mathrm{p}<0.001)$ with the alignment of the knee. There was no significant association of the $\mathrm{T}$ scores with obesity (BMI). Tibial T scores showed a stronger association with alignment than femoral $\mathrm{T}$ scores: correlation coefficients were $-0.52(\mathrm{p}<0.05)$ for the medial tibia, -0.38 (NS) for the medial femur, $+0.76(\mathrm{p}<0.001)$ for the lateral tibia, and +0.31 (NS) for the lateral femur.

When studying the association of patellar cartilage loss with knee alignment, there was no correlation when entering valgus as negative values and varus as positive values into the regression $(r=-0.09)$. When transforming negative (valgus) into positive values and thus treating both forms of malalignment equally (independent of direction), correlation between patellar cartilage loss and static alignment increased to $r=0.30$, but did not reach statistical significance.

\section{DISCUSSION}

In this study we have analysed the magnitude of cartilage loss throughout cartilage plates of the knee in patients with advanced osteoarthritis, before TKA, using quantitative MR imaging technology. In addition, we studied the relation between tibial, femoral, and patellar cartilage loss and valgus/ varus malalignment of the knee in advanced osteoarthritis.

Limitations of this study include the modest sample size and its cross sectional nature. However, to investigate cartilage loss and its relation to alignment longitudinally in advanced osteoarthritis (before knee arthroplasty), observation periods of 10 years and more are required. The strength of the study is that the accuracy of the measurements was confirmed in the same study sample by applying established invasive measurements postoperatively, ${ }^{6}$ and that, in contrast to radiography ${ }^{20}$ quantitative MR imaging allowed us to differentiate tibial, femoral, and patellar cartilage loss accurately in advanced osteoarthritis.

Previous MRI studies have focused on cartilage volume as a quantitative end point. However, cartilage volume scales strongly with bone size and this therefore coincides with a large intersubject variability, both in healthy volunteers and in patients. This variability severely limits the capacity to differentiate effectively between healthy subjects and patients in cross sectional studies. Though cartilage volume can be normalised to body weight, body height, age, and other factors, ${ }^{21}$ the correlation with anthropometric variables is relatively week, ${ }^{22}{ }^{23}$ rendering this approach relatively ineffective. As the individual subchondral bone area (tAB) can be reliably determined from MR image data ${ }^{619}$ and correlates more strongly with cartilage volume than other variables, normalisation to $\mathrm{tAB}$ can be used to effectively enhance $\mathrm{T}$ scores of cartilage morphology for osteoarthritis patients in cross sectional studies. ${ }^{7}$ Note, however, that this requires a different approach to cartilage segmentation, as not only cartilage but also the denuded bone interface area of each cartilage plate needs to be traced in each slice. This tracing must exclude peripheral osteophytes which render the bone interface area larger than it is before the onset of osteoarthritis.

The current data confirm that normalisation to total subchondral bone area is more effective than normalisation 
to other variables such as body weight and height, when trying to discriminate between healthy subjects and patients with osteoarthritis. This applied without exception to all cartilage plates of the knee examined here.

When comparing cartilage loss in different cartilage plates of the knee, we found a substantial amount of heterogeneity and only a weak correlation between femoral and tibial cartilage loss. These findings are important as they clearly suggest that femoral and tibial cartilage plates should be measured separately and that measuring cartilage loss in just one of them is insufficient for estimating cartilage loss in the other. ${ }^{14}$ Cicuttini et $a l^{24}$ described correlation coefficients of around 0.75 between femoral and tibial cartilage volume in healthy subjects and patients with moderate osteoarthritis. They concluded that it may therefore be sufficient to measure only tibial cartilage in femorotibial osteoarthritis. Note that a similar correlation was observed in our current study $(r=0.65$ between medial tibial and femoral cartilage volume), but that the correlation became weaker when cartilage volume was normalised to tAB $(r=0.51)$. This effect is readily explained by the fact that the intersubject variability of cartilage volume was reduced when normalising it to bone size. Our findings suggest that in advanced osteoarthritis only approximately $25 \%$ of the variability in femoral cartilage loss is explained by the cartilage loss measured in the tibia and vice versa, and the correlations are even weaker between the medial and lateral femorotibial compartments, and with the patella. This suggests that the proportion of femoral and tibial cartilage loss is highly variable between osteoarthritis patients, and that it is essential to measure both cartilage plates separately. Other studies have determined an aggregate value for cartilage volume in the tibia and femur. ${ }^{25}{ }^{26}$ As the factors determining the proportion of femoral and tibial cartilage loss in femorotibial osteoarthritis are currently unknown and need to be explored further, we suggest that both cartilage plates should be determined separately.

As expected, we found a significant correlation between femorotibial cartilage loss and malalignment of the knee, and this result is in agreement with previous longitudinal studies employing radiography ${ }^{11}$ and MR imaging. ${ }^{12}$ We cannot determine retrospectively the extent to which malalignment was the cause or effect of cartilage loss, but the range in static alignment of the knee among the subjects was large $\left(14^{\circ}\right.$ varus to $19^{\circ}$ valgus). Also, a recent longitudinal study has confirmed that the degree of malalignment at baseline was associated with prospective cartilage loss in the relevant femorotibial compartments. ${ }^{12}$

An interesting finding of our study is that, in patients with severe osteoarthritis, the correlation of cartilage loss and alignment was greater for the tibia than for the femur. This observation has not been made in patients with moderate osteoarthritis. ${ }^{12}$ Given the moderate sample size, this finding should be interpreted with caution and will need to be confirmed in larger cohorts. However, the current results indicate that malalignment may represent a stronger determinant of tibial than femoral cartilage loss, and this may be a potential reason for the heterogeneity in femoral versus tibial cartilage loss in advanced osteoarthritis.

When considering alignment independent of valgus and varus, there was a weak correlation with patellar cartilage loss. Although the correlation did not reach statistical significance in this sample, it was almost as strong as that between alignment and femoral cartilage loss. These findings indicate that patellar cartilage loss may also be associated with alignment of the knee, potentially because of the higher pressure in the medial or lateral patellar facet. Future studies should thus investigate the cartilage in the medial and lateral facet separately, to determine whether valgus malalignment is specifically correlated to lateral facet cartilage loss, and varus malalignment to medial facet cartilage loss.

In summary, this study shows that quantitative MR imaging is most discriminative between healthy subjects and patients when cartilage volume is normalised to the total subchondral bone area. Cartilage loss was found to be highly variable in patients with advanced osteoarthritis, this also applying to the femur and tibia of the same compartment. Femorotibial cartilage loss was associated with alignment of the knee, but the association was found to be considerably stronger for the tibia than for the femur. The study also provides some evidence that patellar cartilage loss may be weakly associated with alignment, the correlation being of the same order of magnitude as for femoral cartilage. The current data show that, in cross sectional studies, cartilage volume must be normalised to bone interface area to provide useful T scores of cartilage loss in osteoarthritis. Patellar, tibial, and femoral $\mathrm{T}$ scores should be measured separately, due to the larger heterogeneity of tibial cartilage loss in advanced osteoarthritis. Our findings further suggest that the mechanism and cause-effect relation of malalignment and tibial, femoral, and patellar cartilage loss should be examined more closely in larger cohorts of patients with osteoarthritis, using quantitative MR imaging technology.

\section{ACKNOWLEDGEMENTS}

We thank the German Research Society (DFG) for funding this work.

\section{Authors' affiliations}

R von Eisenhart-Rothe, H Graichen, Department of Orthopaedics, University of Frankfurt, Frankfurt, Germany

M Hudelmaier, F Eckstein, Institute for Anatomy and Musculoskeletal Research, Paracelsus Private Medical University, Salzburg, Austria, and Chondrometrics $\mathrm{GmbH}$, Ainring, Germany

T Vogl, Department of Diagnostic Radiology, University of Frankfurt L Sharma, Department of Rheumatology, Northwestern University, Chicago, Illinois, USA

\section{REFERENCES}

1 Yelin E, Callahan LF. The economic cost and social and psychological impact of musculoskeletal conditions. National Arthritis Data Work Groups. Arthritis Rheum 1995;38:1351-62.

2 Creamer P, Hochberg MC. Osteoarthritis. Lancet 1997;350:503-8.

3 Peterfy CG, van Dijke CF, Janzen DL, Gluer CC, Namba R, Majumdar S, et al. Quantification of articular cartilage in the knee with pulsed saturation transfer subtraction and fat-suppressed MR imaging: optimization and validation. Radiology 1994:192:485-91.

4 Burgkart R, Glaser C, Hyhlik-Durr A, Englmeier KH, Reiser M, Eckstein F. Magnetic resonance imaging-based assessment of cartilage loss in severe osteoarthritis: accuracy, precision, and diagnostic value. Arthritis Rheum $2001 ; 44: 2072-7$.

5 Cicuttini F, Forbes A, Morris K, Darling S, Bailey M, Stuckey S. Gender differences in knee cartilage volume as measured by magnetic resonance imaging. Osteoarthritis Cartilage 1999;7:265-71.

6 Graichen H, Eisenhart-Rothe R, Vogl T, Englmeier KH, Eckstein F. Quantitative assessment of cartilage status in osteoarthritis by quantitative magnetic resonance imaging: technical validation for use in analysis of cartilage volume and further morphologic parameters. Arthritis Rheum 2004;50:811-16.

7 Burgkart R, Glaser C, Hinterwimmer S, Hudelmaier M, Englmeier KH, Reiser $M$, et al. Feasibility of $T$ and $Z$ scores from magnetic resonance imaging data for quantification of cartilage loss in osteoarthritis. Arthritis Rheum 2003;48:2829-35.

8 Felson DT, Zhang Y, Hannan MT, Naimark A, Weissman BN, Aliabadi P, et al. The incidence and natural history of knee osteoarthritis in the elderly. The Framingham Osteoarthritis Study. Arthritis Rheum 1995;38:1500-5.

9 Felson DT, Zhang Y. An update on the epidemiology of knee and hip osteoarthritis with a view to prevention. Arthritis Rheum 1998;41:1343-55.

10 Doherty M. Risk factors for progression of knee osteoarthritis. Lancet 2001;358:775-6.

11 Sharma L, Song J, Felson DT, Cahue S, Shamiyeh E, Dunlop DD. The role of knee alignment in disease progression and functional decline in knee osteoarthritis. JAMA 2001;286:188-95.

12 Cicuttini F, Wluka A, Hankin J, Wang Y. Longitudinal study of the relationship between knee angle and tibiofemoral cartilage volume in subjects with knee osteoarthritis. Rheumatology (Oxford) 2004;43:321-4.

13 Sharma L, Lou C, Cahue S, Dunlop DD. The mechanism of the effect of obesity in knee osteoarthritis: the mediating role of malalignment. Arthritis Rheum 2000;43:568-75. 
14 Glaser C, Burgkart R, Kutschera A, Englmeier KH, Reiser M, Eckstein F. Femoro-tibial cartilage metrics from coronal MR image data: technique, testretest reproducibility, and findings in osteoarthritis. Magn Reson Med 2003;50:1229-36.

15 Vanwanseele B, Eckstein F, Knecht H, Spaepen A, Stussi E. Longitudinal analysis of cartilage atrophy in the knees of patients with spinal cord injury. Arthritis Rheum 2003;48:3377-3381.

16 Hinterwimmer S, Krammer M, Krotz M, Glaser C, Baumgart R, Reiser M, et al. Cartilage atrophy in the knees of patients after seven weeks of partial load bearing. Arthritis Rheum 2004;50:2516-20.

17 Stammberger T, Eckstein F, Michaelis M, Englmeier KH, Reiser M. Interobserver reproducibility of quantitative cartilage measurements: comparison of B-spline snakes and manual segmentation. Magn Reson Imaging 1999;17:1033-42.

18 Stammberger T, Eckstein F, Englmeier KH, Reiser M. Determination of 3D cartilage thickness data from MR imaging: computational method and reproducibility in the living. Magn Reson Med 1999;41:529-36.

19 Hohe J, Ateshian G, Reiser M, Englmeier KH, Eckstein F. Surface size, curvature analysis, and assessment of knee joint incongruity with MRI in vivo. Magn Reson Med 2002;47:554-61.

20 Buckland-Wright JC, Macfarlane DG, Lynch JA, Jasani MK, Bradshaw CR. Joint space width measures cartilage thickness in osteoarthritis of the knee: high resolution plain film and double contrast macroradiographic investigation. Ann Rheum Dis 1995;54:263-8.

21 Cicuttini FM, Wluka AE, Forbes A, Wolfe R. Comparison of tibial cartilage volume and radiologic grade of the tibiofemoral joint. Arthritis Rheum 2003;48:682-8.

22 Eckstein F, Winzheimer M, Hohe J, Englmeier KH, Reiser M. Interindividual variability and correlation among morphological parameters of knee joint cartilage plates: analysis with three-dimensional MR imaging. Osteoarthritis Cartilage 2001;9:101-11.

23 Hudelmaier $M$, Glaser C, Englmeier KH, Reiser M, Putz R, Eckstein F. Correlation of knee-joint cartilage morphology with muscle cross-sectional areas vs. anthropometric variables. Anat Rec 2003;270A:175-84.

24 Cicuttini FM, Wluka AE, Stuckey SL. Tibial and femoral cartilage changes in knee osteoarthritis. Ann Rheum Dis 2001;60:977-80.

25 Raynauld JP, Kauffmann C, Beaudoin G, Berthiaume MJ, de Guise JA, Bloch DA, et al. Reliability of a quantification imaging system using magnetic resonance images to measure cartilage thickness and volume in human normal and osteoarthritic knees. Osteoarthritis Cartilage 2003;11:351-60.

26 Raynauld JP, Martel-Pelletier J, Berthiaume MJ, Labonte F, Beaudoin G, de Guise JA, et al. Quantitative magnetic resonance imaging evaluation of knee osteoarthritis progression over two years and correlation with clinical symptoms and radiologic changes. Arthritis Rheum 2004;50:476-87.

\section{bmjupdates+}

bmjupdatest is a unique and free alerting service, designed to keep you up to date with the medical literature that is truly important to your practice.

bmjupdates+ will alert you to important new research and will provide you with the best new evidence concerning important advances in health care, tailored to your medical interests and time demands.

Where does the information come from?

bmjupdates+ applies an expert critical appraisal filter to over 100 top medical journals A panel of over 2000 physicians find the few 'must read' studies for each area of clinical interest

Sign up to receive your tailored email alerts, searching access and more...

www.bmjupdates.com 